\section{Single-molecule imaging of hyaluronan in human synovial fluid}

\author{
Joachim Kappler, a,* Tim P. Kaminski, a,b \\ Volkmar Gieselmann, ${ }^{a}$ Ulrich Kubitscheck, ${ }^{b}$ and Jörg \\ Jerosch $^{\mathrm{c}}$ \\ a Rheinische Friedrich-Wilhelms-Universität Bonn, Institut für \\ Biochemie und Molekularbiologie, Nussallee 11, 53115 Bonn, \\ Germany \\ ${ }^{\mathrm{b}}$ Institut für Physikalische und Theoretische Chemie, Wegelerstraße \\ 12, 53115 Bonn, Germany \\ 'Klinik für Orthopädie, Unfallchirurgie und Sportmedizin, \\ Johanna-Etienne-Krankenhaus, Am Hasenberg 46, 41462 Neuss, \\ Germany
}

\begin{abstract}
Human synovial fluid contains a high concentration of hyaluronan, a high molecular weight glycosaminoglycan that provides viscoelasticity and contributes to joint lubrication. In osteoarthritis synovial fluid, the concentration and molecular weight of hyaluronan decrease, thus impairing shock absorption and lubrication. Consistently, substitution of hyaluronan (viscosupplementation) is a widely used treatment for osteoarthritis. So far, the organization and dynamics of hyaluronan in native human synovial fluid and its action mechanism in viscosupplementation are poorly characterized at the molecular level. Here, we introduce highly sensitive single molecule microscopy to analyze the conformation and interactions of fluorescently labeled hyaluronan molecules in native human synovial fluid. Our findings are consistent with a random coil conformation of hyaluronan in human synovial fluid, and point to specific interactions of hyaluronan molecules with the synovial fluid matrix. Furthermore, single molecule microscopy is capable of detecting the breakdown of the synovial fluid matrix in osteoarthritis. Thus, single molecule microscopy is a useful new method to probe the structure of human synovial fluid and its changes in disease states like osteoarthritis. ( $\odot 2010$ Society of Photo-Optical Instrumentation Engineers. [DOI: 10.1117/1.3514639]
\end{abstract}

Keywords: single molecule microscopy; hyaluronan; synovial fluid; osteoarthritis; viscosupplementation.

Paper 10472LR received Aug. 25, 2010; revised manuscript received Oct. 15, 2010; accepted for publication Oct. 18, 2010; published online Nov. 23, 2010.

Single molecule microscopy is a recent development in the field of biomedical optics that allows tracking of the motion of individual fluorescent biomolecules with a time resolution in the millisecond range at a localization accuracy down to $15 \mathrm{~nm} .{ }^{1}$ Analysis of trajectory data reveals information on the conformation of biomolecules, and can be used to probe their interaction with their environment.

Hyaluronan (HA) is a glycosaminoglycan that is abundant in synovial fluid, where its viscoelastic and shock-absorbing properties are important for joint function. ${ }^{2}$ In synovial fluid

\footnotetext{
*Address all correspondence to: Joachim Kappler, Institut für Biochemie und Molekularbiologie, Nussallee 11, D-53115 Bonn, Germany. Tel: 49-228734744; Fax: 49-228-732416. E-mail: kapplerj@uni-bonn.de.
}

from osteoarthritis patients, the concentration and molecular weight of HA are reduced. ${ }^{2,3}$ Injection of HA or cross-linked HA into the synovial fluid in osteoarthritis relieves pain and improves joint function in the long term. ${ }^{4,5}$ This widely used treatment is termed viscosupplementation. The viscoelasticity of HA molecules can be explained in terms of a random coil polymer model, ${ }^{6}$ but direct observation of single HA molecules in solution to confirm this concept was difficult in the past due to technical limitations. Recently, single molecule microscopy became available to examine the conformation and mobility of HA in solution. ${ }^{1}$ This method confirmed that HA molecules form random coil structures in buffer solution. ${ }^{7}$ In tissues, however, more stably organized forms of HA like cable- and fiber-like structures can be found. ${ }^{8}$ Here, we addressed if it is possible to examine the conformation and interactions of HA molecules in native human synovial fluid using single molecule microscopy.

The study was approved by the ethics committee of the University of Bonn, Germany, and informed consent was obtained from the patients. Synovial fluid was collected from a 69-yearold male patient with a meniscus lesion undergoing arthroscopy (SF1) and from a 76-year-old female patient with advanced osteoarthritis undergoing knee joint replacement surgery (SF2).

Single-particle imaging experiments were performed using a custom-built single molecule microscope based on a Zeiss Axiovert 200TV equipped with an $63 \times$ NA 1.4 oil immersion objective lens (Carl Zeiss Microimaging GmbH, Jena, Germany). Fluorescence was excited at $532 \mathrm{~nm}$ by a diode-pumped solid state laser (Cobolt Dual Calypso, Solna, Sweden). Laser illumination was switched on only during image acquisition by means of an acousto-optical tunable filter (AA Optoelectronics, Orsay Cedex, France). For single-particle image acquisition, we used the iXon DV-860 BI camera (Andor Technology, Belfast, Northern Ireland) in combination with a $4 \times$ magnifier, yielding a pixel size in the object space of $95.24 \mathrm{~nm}$. Generally, 2500 frames were recorded in a single movie, with a frame rate of $k_{\text {acq }}=100 \mathrm{~Hz}$. All measurements were performed at $22^{\circ} \mathrm{C}$. Since synovial joints are avascular compartments, many of which are located in the periphery of the body, it seems reasonable to assume that the temperature in peripheral joints exposed to the ambient can be substantially lower than $37^{\circ} \mathrm{C}$. The temperature of $22^{\circ} \mathrm{C}$ was meant to approximate the conditions in peripheral joints. Fluorescent HA (HA-TRITC) was prepared from HA from rooster comb (average molecular weight $1.5 \mathrm{MDa}$ ) as described with modifications, ${ }^{7}$ and used at a concentration of $\approx 170 \mathrm{pM}$ at a molecular weight basis. As an inert macromolecular tracer fluorescent, 200-kDa dextran was used.

When TRITC-labeled HA was added to small volumes of synovial fluid samples (ca $5 \mu$ l per measurement) and imaged, single TRITC-labeled HA molecules were clearly detected, although the background fluorescence signal of synovial fluid was higher than that of buffer solutions [Figs. 1(a) and 1(b)]. HA-TRITC molecules were mobile and had rounded shapes as expected for random coils [Fig. 1(a)]. At no stage were immobile organized forms of HA observed.

For tracking single HA molecules in equilibrated samples, identification of the single molecule signals was accomplished using Diatrack 3.01 (Semasopht, North Epping, Australia),

1083-3668/2010/15(6)/060504/3/\$25.00 @ 2010 SPIE 


\section{JBO LETTERS}

(a)

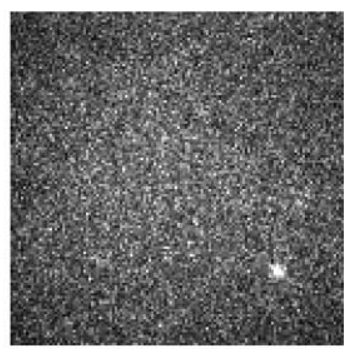

(c)

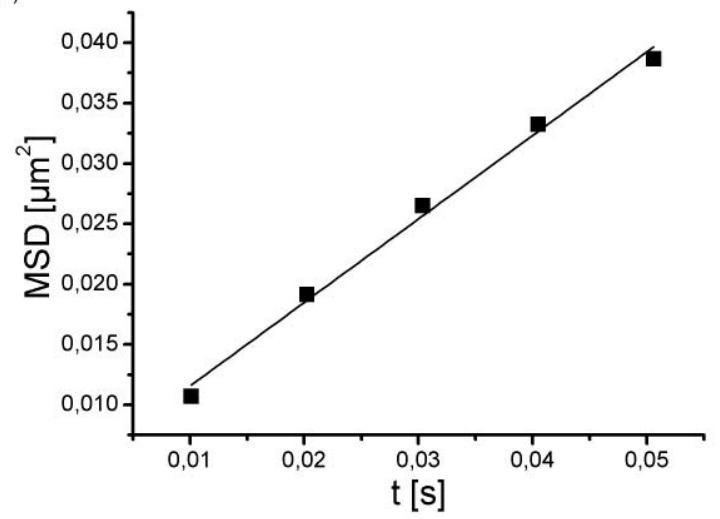

(b)

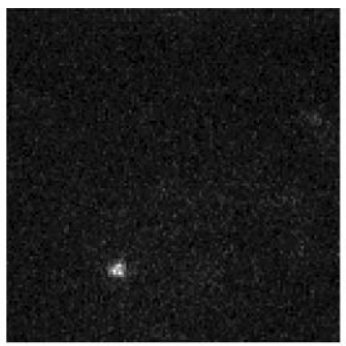

(d)

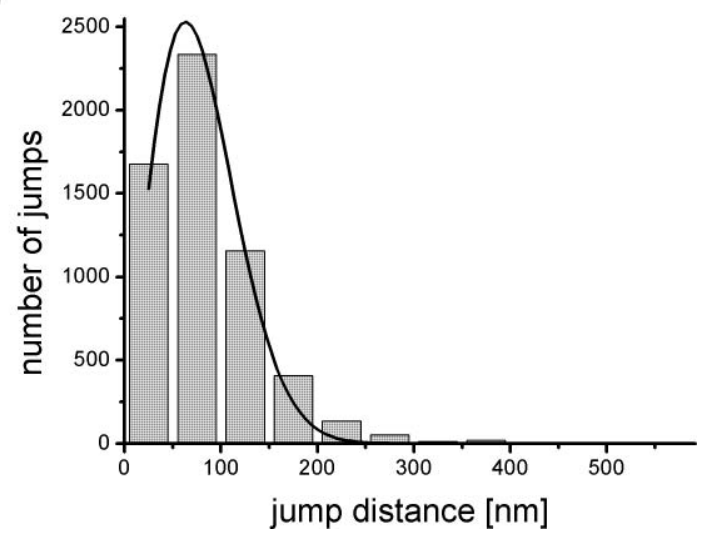

Fig. 1 (a) Shape of hyaluronan-TRITC molecules in human synovial fluid. The object field size shown is $12.2 \times 12.2 \mu \mathrm{m}$. (b) HA-TRITC molecules in buffer solution. Field size is as in (a). (c) Linear dependence of mean-square displacements of hyaluronan-TRITC molecules in synovial fluid (SF1). Filled symbols represent mean values of the mean-square displacements measured at different times after mixing HA-TRITC, as indicated in the inset. SEMs were below 5\%. The straight line represents a linear fit of the mean values. (d) Jump distance distribution of HA-TRITC molecules in synovial fluid. The black line represents a best-fit approximation of the data, assuming Brownian motion of HA-TRITC with $D=0.18559 \mu \mathrm{m}^{2} / \mathrm{s}$.

a commercial image processing program for the analysis of single-particle signals as described in Ref. 7. From the trajectory data we calculated the mean-square displacement (MSD) over time and the jump distance distribution to obtain diffusion coefficients as described previously. ${ }^{7}$

Analysis of the trajectories revealed Brownian motion of HA-TRITC in the synovial fluid from a patient with a meniscus lesion without prior history of osteoarthritis (SF1), as indicated by the linear increase of the MSD over time [Fig. 1(c)]. In equilibrium, the diffusion coefficient of HA-TRITC in synovial fluid was $\approx 0.19 \mu \mathrm{m}^{2} / \mathrm{s}$ according to a best-fit approximation of the global jump distance distribution, assuming Brownian motion of HA-TRITC [Fig. 1(d)]. This latter value was $\approx 13$-fold smaller than the diffusion coefficient of HA-TRITC in buffer solution (Fig. 2). In contrast, the diffusion coefficient of the macromolecular tracer dextran in the same human synovial fluid (SF1) was only $\approx 7$-fold smaller than in buffer solution (Fig. 2). Thus, HA-TRITC interacted more strongly with the synovial fluid environment than dextran. This contrasted markedly with our observations using synovial fluid from a patient with advanced osteoarthritis (SF2). In osteoarthritis synovial fluid, the diffusion coefficient of HA-TRITC was only $\approx 3$-fold smaller than in buffer solution (Fig. 2). Interestingly, this decrease was comparable to the $\approx 4$-fold decrease of the diffusion coefficient of dextran in osteoarthritis synovial fluid in comparison to buffer solution. Furthermore, dextran diffused considerably faster in osteoarthritis synovial fluid than in synovial fluid from the patient without history of osteoarthritis. To confirm that the dif- ferences of the diffusion coefficients in the two synovial fluids were significant, for each individual movie file jump distance distributions were fitted separately. In all cases the measured diffusion coefficients were consistent with a Gaussian distribution $(p<0.05)$, as expected for equilibrated samples. By this procedure the following mean values of the diffusion coefficients $\left( \pm\right.$ SEM) were obtained (all in $\mu \mathrm{m}^{2} / \mathrm{s}$ ): dextran in SF1 (control): $D=0.92 \pm 0.05$ ( $n=17$ movies); dextran in SF2 (advanced osteoarthritis): $D=1.20 \pm 0.17$ ( $n=18$ movies); HA-TRITC in SF1: $D=0.19 \pm 0.01$ ( $n=26$ movies $)$; and HA-TRITC in SF2: $D=0.99 \pm 0.07$ ( $n=45$ movies). That these numbers differed from the values obtained by global fitting (Fig. 2) is most likely explained by heterogeneity of the movies with respect to the number of jumps. This random heterogeneity was not taken into account when the means were calculated. Furthermore, fitting errors cumulate in this analysis. Thus, these values are less accurate than the values obtained by global fitting (Fig. 2). Comparison of the mean values using the $t$-test revealed that the difference of the diffusion coefficients of HA-TRITC between SF1 and SF2 was highly significant $(p<0.01)$. Moreover, the difference of the diffusion coefficients of dextran between SF1 and SF2 was highly significant $(p<0.01)$. Taken together, these findings were consistent with a degenerative change of the synovial fluid matrix in osteoarthritis that led to the loss of specific interactions with HA-TRITC.

Our experiments show that single molecule microscopy observation of HA-TRITC and of tracer molecules in human synovial fluid samples as obtained during routine joint diagnostics 


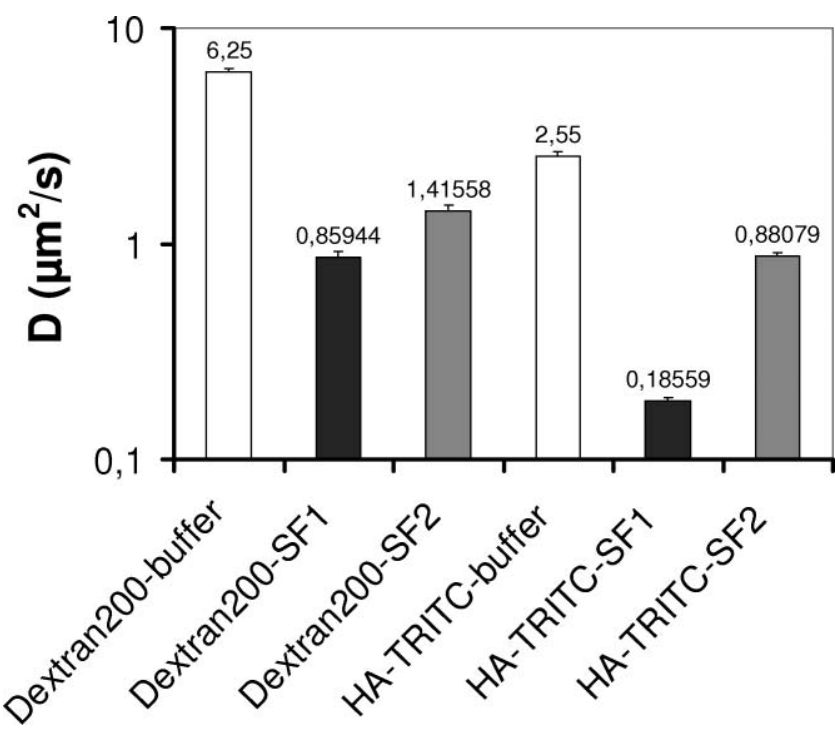

Fig. 2 Equilibrium diffusion coefficients of hyaluronan and a macromolecular tracer (dextran 200) in human synovial fluid. Bars represent the diffusion coefficient of fluorescent hyaluronan (HA-TRITC) or dextran, respectively, as determined by fitting the global jump distance distributions with a one-component model in buffer solution (open bars), in synovial fluid from a patient with a meniscus lesion (SF1, black bars), and in synovial fluid from a patient with advanced osteoarthritis (SF2, gray bars). Error bars represent the standard errors of the fits as indicated in Origin (version 8). The number of jumps analyzed were: dextran in buffer, 974; dextran in SF1, 4175; dextran in SF2, 3331; and HA-TRITC in buffer, 2819; HA-TRITC in SF1, 5813; and HA-TRITC in SF2, 3794. For statistical comparisons, individual movies were analyzed separately (see text).

is readily feasible with minimal (microliter) amounts of synovial fluid, and yield accurate quantitative measurements of diffusion coefficients.

The hydrodynamic diameter HA molecules of $1.500 \mathrm{kDa}$ is $\approx 150 \mathrm{~nm},{ }^{7}$ close to the optical resolution of our microscope of $\approx 250 \mathrm{~nm}$. Thus, aggregates of such molecules or more organized forms of HA, for example the micrometer-sized structures seen in atomic force images, ${ }^{6}$ would have been resolved by our method. From the rounded shapes of HA molecules in synovial fluid, the absence of stable aggregates or organized forms, and their noncompacted conformation according to their large hydrodynamic radius, we conclude that HA forms random coils in human synovial fluid [Fig. 1(a)].

In contrast to the diffusion of HA-TRITC in buffer solution, ${ }^{7}$ the mobility of HA-TRITC in synovial fluid was substantially lower with $\approx 13$-fold lower diffusion coefficient in SF1 (Fig. 2). This suggests that HA-TRITC undergoes molecular interactions within synovial fluid. Since the diffusion coefficient of the inert tracer molecule dextran in synovial fluid is diminished to a considerably smaller extent, HA-TRITC seems to undergo specific interactions within the synovial fluid environment (Fig. 2). The physicochemical character of these interactions of HA very likely is heterogeneous. On one hand, physical entanglements between the highly flexible HA chains are likely to play a role, ${ }^{6}$ as described for concentrated HA solutions., ${ }^{910}$ Such entanglements are less likely to occur for the smaller dextran tracer molecules, because these are subject to less mutual crowding. On the other hand, specific interactions with HA-binding protein domains may play a role. Thus, our observations are in agreement with the concept that in human synovial fluid, a matrix exists that specifically integrates randomly coiled HA molecules into a dynamic supramolecular structure. The strong viscoelasticity of synovial fluid is consistent with such a type of organization. The degenerative loss of this matrix in osteoarthritis facilitates the diffusion of HA-TRITC and of the tracer dextran, and abolishes the specific interactions of HA-TRITC with synovial fluid (Fig. 2).

In conclusion, single molecule microscopy is capable of detecting the loss of the HA-based network structure in synovial fluid in osteoarthritis, which reduces its viscosity and hydrodynamic lubricant properties. Thus, single molecule microscopy is a useful method for probing the organization of human synovial fluid and to examine its changes in disease states like osteoarthritis.

\section{References}

1. D. Grünwald, A. Hoekstra, T. Dange, V. Buschmann, and U. Kubitscheck, "Direct observation of single protein molecules in aqueous solution," Chemphyschem. 7(4), 812-815 (2006).

2. H. Fam, J. T. Bryant, and M. Kontopoulou, "Rheological properties of synovial fluids," Biorheology 44(2), 59-74 (2007).

3. P. Ghosh and D. Guidolin, "Potential mechanism of action of intraarticular hyaluronan therapy in osteoarthritis: are the effects molecular weight dependent?" Semin. Arthritis Rheum. 32(1), 10-37 (2002).

4. N. Bellamy, J. Campbell, V. Robinson, T. Gee, R. Bourne, and G. Wells, "Viscosupplementation for the treatment of osteoarthritis of the knee," Cochrane Database Syst. Rev., CD005321 (2005).

5. K. M. Jordan, et al., "EULAR Recommendations 2003: an evidence based approach to the management of knee osteoarthritis: report of a task force of the Standing Committee for International Clinical Studies Including Therapeutic Trials (ESCISIT)," Ann. Rheum. Dis. 62(12), 1145-1155 (2003).

6. M. K. Cowman and S. Matsuoka, "Experimental approaches to hyaluronan structure," Carbohydr. Res. 340(5), 791-809 (2005).

7. T. Kaminski, J. P. Siebrasse, V. Gieselmann, U. Kubitscheck, and J. Kappler, "Imaging and tracking of single hyaluronan molecules diffusing in solution," Glycoconj. J. 25(6), 555-560 (2008).

8. C. A. de la Motte, V. C. Hascall, J. Drazba, S. K. Bandypadhyay, and S. A. Strong, "Mononuclear leukocytes bind to specific hyaluronan structures on colon mucosal smooth muscle cells treated with polyinosinic acid:polycytidylic acid: inter-alpha-trypsin inhibitor is crucial to structure and function," Am. J. Pathol. 163(1), 121-133 (2003).

9. P. Gribbon, B. C. Heng, and T. E. Hardingham, "The molecular basis of the solution properties of hyaluronan investigated by confocal fluorescence recovery after photobleaching," Biophys. J. 77(4), 2210-2216 (1999).

10. P. Gribbon, B. C. Heng, and T. E. Hardingham, "The analysis of intermolecular interactions in concentrated hyaluronan solutions suggest no evidence for chain-chain association," Biochem. J. 350, 329-335 (2000). 